\title{
Researches on a practical system for concentration detection of human exhaled $\mathrm{CO}_{2}$ gas
}

\author{
Rong-Guo Yan $^{1}$, Bin Ge ${ }^{1}$, Hai-Ming Xie ${ }^{1}$
}

School of medical instrument and food engineering, University of Shanghai for Science and Technology, Shanghai 200093. Correspondence should be addressed to Rong-Guo Yan (yanrongguo@usst.edu.cn), Tel: +862155271115.

Received July $30^{\text {th }}, 2008$; revised November $12^{\text {th }}, 2008$; accepted November $19^{\text {th }}, 2008$

\begin{abstract}
In clinics, especially in the emergency department, carbon dioxide $\left(\mathrm{CO}_{2}\right)$ is considered as the sixth vital sign in evaluating a patient's medical status. However, its wide applications in developing countries are dissatisfactory due to their high cost compared to their economic growth. In order to develop a practical system for concentration detection of human exhaled $\mathrm{CO}_{2}$ gas for our own, we studied the detection principle based upon the non-dispersive infrared (NDIR) measuring technique and related LambertBeer's equation carefully, gave out our functional circuit design of the system, and provided the corresponding graphical user interface (GUI) for $\mathrm{CO}_{2}$ detection. Finally, the experiment shows that it may be a practical system, and will give benefits for the development of our medical care in the future.
\end{abstract}

Keywords: $\mathrm{CO}_{2}$ Concentration Detection, NDIR, Lambert-Beer's Equation, Detection Module, Instrument Module

\section{INTRODUCTION}

As we know, carbon dioxide $\left(\mathrm{CO}_{2}\right)$, as a gas byproduct produced by human cellular metabolism and finally exhaled out into the air through our noise, can be measured to reflect the systematic functions of metabolism, circulation and ventilation $[1,2,3]$. Especially, through the noninvasive monitoring of end-tidal carbon dioxide $\left(\mathrm{ETCO}_{2}\right)$, which is also known as capnography measuring the maximum carbon dioxide level reached at the end of each breath $[4,5,6]$, it can provide the doctor or the nurse the sixth vital sign, as important as five other signs, i.e., respiration, heart rate, temperature, blood pressure and pain in evaluating the medical status of the subjects. In America, capnography, other than monitoring pulse oxygen saturation, has already been considered as one of

The project is supported by the research award fund for selectively culturing excellent young teachers in higher educations in shanghai (grant No. slg-07045). the "basic standards" for monitoring for all the subjects receiving anesthesia.

The most common principles for $\mathrm{CO}_{2}$ detection are based upon non-dispersive infrared (NDIR) measurement or based upon chemical reactions. Commonly, chemical $\mathrm{CO}_{2}$ gas sensors with sensitive layers have the principal advantage of very low energy consumption and can be reduced in size to fit into microelectronic based systems. However, compared with the NDIR measurement principle [7], chemical $\mathrm{CO}_{2}$ sensors face major obstacles like short-and long term drift effects and a rather low overall lifetime $[8,9]$.

Although the technology carried out for monitoring expired $\mathrm{CO}_{2}$ gas based upon NDIR is not a new technology $[10,11,12]$, its wide applications, such as in mechanical ventilator, anesthesia machine, and etc., in developing countries are still left in a dissatisfactory condition due to its high cost compared to their economic growth. However nowadays, with the development of integrated circuits and non-dispersive infrared (NDIR) sensing technology, its utilization by clinicians outside the operating room or anesthesia realm has also grown, especially in the emergency department.

Thus, this paper will present the principle for $\mathrm{CO}_{2}$ gas concentration measurement; introduce its functional circuit design that we applied; and provide its current experimental result to show its practicability for use.

\section{PRINCIPLE FOR DETECTION}

The method we used for concentration detection of $\mathrm{CO}_{2}$ gas is based upon the NDIR measuring technique and a so-called well-known Lambert-Beer's equation [13]. The equation is given as follow:

$$
I=I_{0} * e^{-k c l}
$$

Where,

$I_{0}=$ the intensity of light incident on the sample;

$I=$ the measured intensity of light after the sample;

$k=$ the absorption coefficient of the analyte gas at the characteristic wavelength $\left(\mathrm{cm}^{2}\right)$;

$c=$ the concentration of the analyte gas $\left(1 / \mathrm{cm}^{3}\right)$;

$l=$ the path-length or the distance that the light traverses the sample gas $(\mathrm{cm})$.

Let 


$$
A=-\ln \left(\frac{I}{I_{0}}\right)
$$

Then according to equation (1), there exists,

$$
A=k c l
$$

In the equation (3), $A$ is expressed in terms of an absorbance. As we know, the parameter $l$ is already determined when the detection system is designed, and the parameters $A$ and $k$ can be measured in the experiment, then $\mathrm{CO}_{2}$ gas concentration $c$ can be calculated accordingly.

As Figure 1 shows, the chamber is made of aluminum; the left-side inner surface adopts arc shape, being specially polished and shot-blasted to increase the intensity onto the right-side detector.

And, basically the concentration detection system for $\mathrm{CO}_{2}$ gas consists of the following four components including: (1) a pulsed infrared (IR) light source at the left end of the chamber or sample room, whose excitation frequency is about $1 \mathrm{~Hz}$ to provide a non-dispersive infrared flashing source; (2) an airway chamber having $l$ path length with a gas inlet and a gas outlet at the up side edge as Figure 1 shows. The gas inlet is connected to our nose with a nose-oxygen-tube like pipe, while the gas outlet gives a way for $\mathrm{CO}_{2}$ gas flowing out of the chamber, and the chamber will be full of $\mathrm{CO}_{2}$ gas when performing detection; and (3) an IR detector at the right end of the chamber. The detector is in fact a dual channel PerkinElmer thermopile sensor housing with two window openings (channel T1 and T2)-each window specialized to be equipped with band-pass filters for gas detection [14]. One optical window opening (T2) senses light at a specific wavelength of $4.26 \mu \mathrm{m}$, a predominant absorption band of $\mathrm{CO}_{2}$ gas (see Figure 2), while the other channel (T1) actually carries a band-pass wavelength centered at $4.0 \mu \mathrm{m}$, other than $\mathrm{CO}_{2}$ gas absorption band at $4.26 \mu \mathrm{m}$, acting as reference [14]. Thus except ground signal, three other signals that the thermopile detector gives are a reference signal (Ref_sig. in Figure 1) for reference use, a real measured signal (Msd sig. in Figure 1), and a temperature signal (Tmp_sig. in Figure 1) giving the real temperature inside the thermopile sensor for later temperature compensation use. Basically, these millivolts $(\mathrm{mV})$ level signals should be amplified, conditioned via a detection module, and then the actual $\mathrm{CO}_{2}$ concentration can be calculated according to the Lambert-Beer's equation by the MCU in an instrument module. Functions of the detection module and the instrument module are described in section 3 .

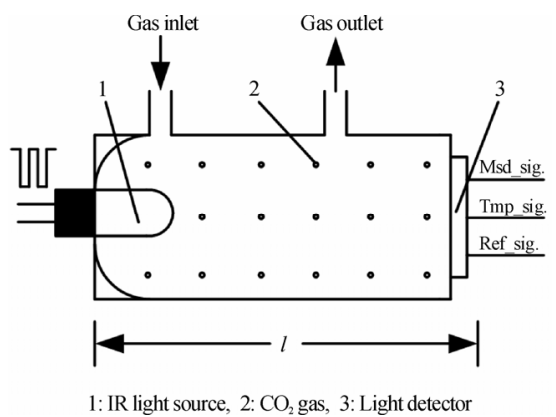

Figure 1. NDIR optical detection system

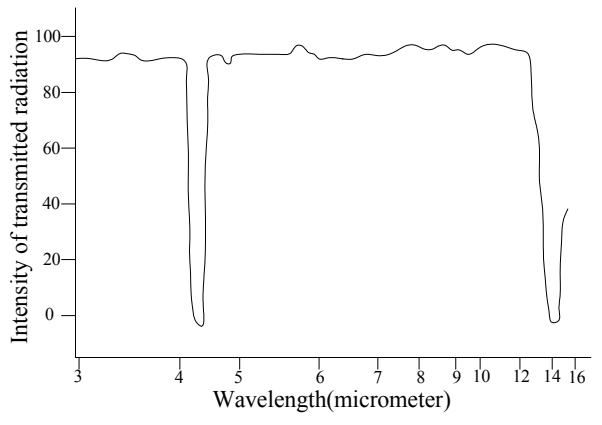

Figure 2. Optical spectral absorption bands of $\mathrm{CO}_{2}, 4.26 \mu \mathrm{m}$ is its predominant absorption peak, and the IR light in between this narrowband can pass the interference filter

\section{FUNCTIONAL CIRCUIT DESIGN}

To accomplish $\mathrm{CO}_{2}$ gas concentration detection as the principle indicates, we designed a practical detection system including two functional modules: the detection module and the instrument module.

\subsection{The Detection Module}

The detection module performed signal amplification, conditioning and voltage following of four signals that are needed to estimate gas concentration of exhaled $\mathrm{CO}_{2}$ as the Lambert-Beer's equation indicates, including one room temperature signal (Room sig.) and three other signals from the thermopile detector, i.e., a reference signal (Ref_sig.), a real measured gas concentration output (Msd_sig.) and a temperature output (Tmp_sig.) for later temperature compensation (See section 2). Finally, these four signals will be provided for the instrument module for calculation.

\subsection{The Instrument Module}

The instrument module mainly performs (1) analog-todigital (A/D) conversion of four signals including a Room_sig., a Ref_sig., a Msd_sig., and a Tmp_sig. from the detection module; (2) calculation gas concentration according to the Lamber-Beer's equation; (3) concentration display on the liquid crystal display (LCD) of the acquired $\mathrm{CO}_{2}$ gas at the sample time; (4) giving warnings and alarms; (5) configuration by a master PC using a RS-232C universal serial interface. Thus, the main components in the instrument module are: (1) an ATmega16L micro controller unit (MCU), which is a high-performance, low-power $\mathrm{AVR}^{\circledR}$ 8-bit microcontroller with an advanced reduced instruction set computer (RISC) architecture and 16KB in-system programmable flash program memory [15]. The MCU is good for programming and debugging using $\mathrm{C}$ language; (2) a master PC performing to finish configuration of the system, such as serial number information, warning limits (upper and lower limit) of gas concentration, calibration and temperature compensation information, and etc., through the RS-232C interface; (3) a DS1307 real time clock (RTC), which is a low-power, full binary-coded decimal 
clock/calendar. Address and data are transferred serially through a two-wire interface (TWI), bidirectional bus that Atmega16L provides. The current time can be set through the RS-232C based graphical user interface written in computer advanced languages, Visual $\mathrm{C}++$, MATLAB, and etc., for example; (4) a beeper and a HF12232F-based LCD, which dynamically gives current time, gas concentration information at current time, and warnings, as well as other information that can be visually prompted on the LCD. The LCD interfaces with the MCU through the serial peripheral interface (SPI) interface that the Atmeag16L provides. (5) an A/D conversion of four signals from the detection module, including one room temperature signal (Room_sig.) and three signals from the thermopile, i.e., a reference signal (Ref_sig.), a real measured output (Msd_sig.) and a temperature output (Tmp_sig.) for later temperature compensation use. The functions of the instrument module can be seen in Figure 3.

\section{RESULTS}

Figure 4 is a graphical user interface (GUI) for $\mathrm{CO}_{2}$ concentration detection, which is written in MATLAB. MATLAB is selected in the paper for its simple programming, rich graphic facilities, built-in functions, and extensive toolboxes. This is especially suitable for engineering professionals like us. From the GUI, we can select serial port (COM1, COM2, and etc.) and baud rate (9600bps, 115200bps, and etc.) to establish communications between the master PC and the Atmeaga16L MCU to perform gas concentration detections using the button SendFF, where hexadecimal $0 x F F$ is used as a handshaking string between two CPUs. Moreover, the button Load Data can load any related saved data. In the graphical window, the real measured output (corresponding to Msd_sig.) and its reference output (corresponding to Ref_sig.) in an experimental test are plotted with different signs. Their minimal value, maximal value, and averaged peak-to-peak difference in the sample period are given in the right frame box.

In order to eliminate errors from intensity variation of the light, and to decrease sensor drift due to temperature variations, the ratio between peak-to-peak difference of the real measured output (Msd_sig.) and the reference output (Ref_sig.) is calculated, and this value is proportional to the current gas concentration. Actually, a relation curve between this ratio and the gas concentration has already established when performing calibration of the system at a discrete concentration step, and has been stored

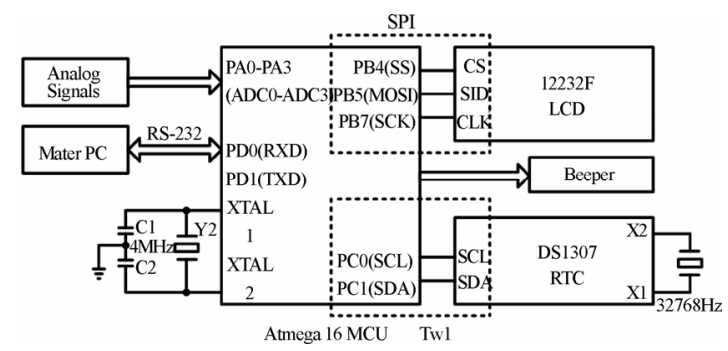

Figure 3. The instrument module

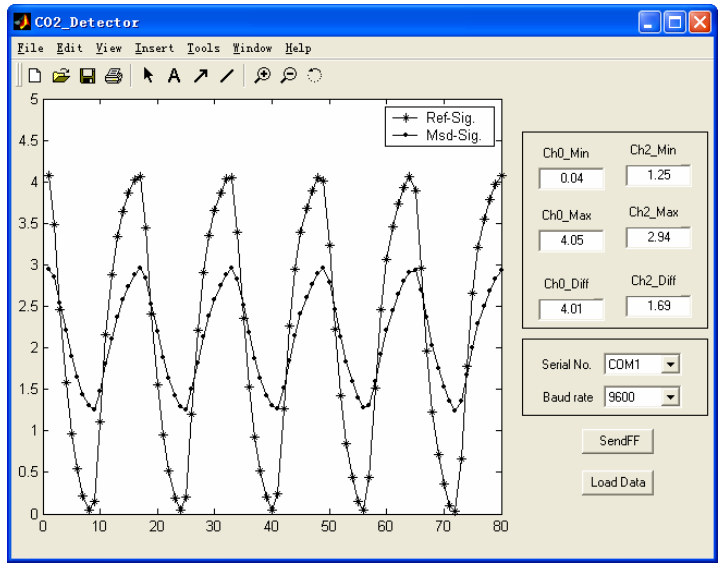

Figure 4. GUl for $\mathrm{CO}_{2}$ detection written in MATLAB

in the EEPROM inside the MCU. The measured $\mathrm{CO}_{2}$ gas concentration can then be calculated using the ordinary least-square interpolation method in between two neighboring discrete concentration values designed in calibration period.

As a human machine interface, the calculated real concentration is also shown on the LCD.

\section{FUTURE WORKS}

In the paper, we introduced the method of concentration detection for human exhaled $\mathrm{CO}_{2}$ gas, described the functional module (the detection module and the instrument module), and provided the GUI for $\mathrm{CO}_{2}$ detection written in MATLAB.

The experiment result shows that it is a practical system for medical $\mathrm{CO}_{2}$ concentration detection whose measurement range is from 0 to $3000 \mathrm{ppm}$ (part per million; $1000 \mathrm{ppm}=0.1 \%$ ) with $\pm 5 \%$ of reading accuracy as desired, and it will give benefits for the development of our medical care in the future, as expected.

However, there are still remaining challenges ahead of us before real practical use. Firstly, the characteristics of the system need to be verified for relatively long time. Secondly, a face mark for the patients receiving supplemental oxygen should be considered in the mechanical design. To provide additional functions, such as remote control of the system are also needed to be considered in the future for specific environments.

\section{REFERENCES}

[1] J. S. Gravenstein, D. A. Paulus, T. J. Hayes (1989) Clinical indications, em: Gravenstein JS, Paulus DA, Hayes TJ-Capnography in clinical practice. Butterworth Publishers, Boston.

[2] M. S. Bhende (1999). Capnography in the pediatric emergency department. Pediatric Emergency Care, 15:64-69.

[3] A. B. Sanders (1989). Capnometry in emergency medicine. Annals of Emergency Medicine, 18:1287-1290.

[4] R. N. N. Gilboy, R. Michael, C. R. N. A. Hawkins, MS (2006). Noninvasive monitoring of end-tidal carbon dioxide in the emergency department. Advanced Emergency Nursing Journal, 28(4): $301-313$.

[5] M. S. Bhende (2001). End-tidal carbon dioxide monitoring in pediatrics: concepts and technology. Journal of Postgraduate Medicine. 47(2): 153-156. 
[6] A. B. Sanders, K. B. Kern, C. W. Otto, M. M. Milander and G. A. Ewy (1989). End-tidal carbon dioxide monitoring during cardiopulmonary resuscitation: a prognostic indicator for survival. The Journal of the American Medical Association. 262 (10): 1347-1351.

[7] Th. Lang, H. D. Wiemhöfer and W. Göpel, Conf. Proc. Eurosensors IX, Stockholm (S) (1995); Carbonate based $\mathrm{CO}_{2}$ sensors with high performance, Sensors and Actuators B, 34, 1996, 383-387.

[8] http://en.wikipedia.org/wiki/CO2_sensor.

[9] Reliable $\mathrm{CO}_{2}$ sensors based with silicon-based polymers on quartz microbalance transducers, R. Zhou, S. Vaihinger, K. E. Geckeler and W. Göpel, Conf. Proc. Eurosensors VII, Budapest
(H) (1993); Sensors and Actuators B, 18-19, 1994, 415-420.

[10] T. Ahrens \& C. Sona, (2003). Capnography application in acute and critical care. AACN Clinical Issues, 14 (2), 123-132.

[11] K. Bhavani-Shankar, (2006). Capnography: A comprehensive educational website. Retrieved July 23, 2006

[12] M. Eichbrecht, (2000, December/2001, January). Capnography: The ventilation vital sign. Retrieved August 2, 2006.

[13] J. D. J. Ingle and S. R. Crouch, Spectrochemical Analysis, Prentice Hall, New Jersey.

[14] http://www.perkinelmer.com/.

[15] http://www.atmel.com/. 